\title{
Low Temperature Casting of Graphene into Various 3-D Shapes
}

\author{
Shu Wan, Hengchang Bi, KuiboYin, Xiao Xie, Yilong Zhou, Neng Wan, Feng Xu, Litao Sun* \\ SEU-FEI Nano-Pico Center, Key Laboratory of MEMS of Ministry of Education, Southeast University, Nanjing, China \\ *Corresponding Author: Litao Sun, slt@,seu.edu.cn
}

\begin{abstract}
Here we report a pH-mediated hydrothermal reduction method that combined with moulding methods, realizing fabrication of ultrahigh density graphene macrostructures with various shapes. This 'compact graphene' (CG) has a compressive strength of $361 \mathrm{MPa}$ (6 times higher than conventional graphite products) and an excellent electrical conductivity. The processing steps are scalable and may contribute to new products. CG structures may be an ideal irradiation protection material for aerospace, atomic energy, and nuclear science due to its high density.
\end{abstract}

Keywords-graphene; compact graphene; casting; high density

\section{INTRODUCTION}

Graphene, a 2-dimensional, single-atom-thick layer of carbon, has attracted attention due to its fascinating properties such as high carrier mobility [1-3], high thermal conductivity $[4,5]$, extraordinary elasticity and stiffness [6], and other properties. While mechanical exfoliation, liquid exfoliation [7], and epitaxial growth [8] can produce pristine graphene, the yields are currently too low for large-scale production of macrostructures. In contrast, chemical reduction of graphene oxide provides "graphene" sheets in massive scale for graphene macrostructures [9-11]. Graphene-based macrostructures prepared to date have been relatively weak mechanically, given their flexible and often relatively porous or open structures, when compared with commercial graphite products. Achieving highly compacted and thus "fully dense" macrostructures based on graphene and measuring the physical properties of such material(s) is thus an important goal.

We present a new method to obtain high-density compact 3D graphene macrostructures (' $C G$ ') with various shapes, using a template-directed hydrothermal method from graphene oxide suspensions. The proposed method has many advantages compared to conventional strategies for forming $3 \mathrm{D}$ shapes with graphite powder, e.g., forming of the desired macrostructure in one step, low-cost, time- and energy-saving, and low-temperature processing $\left(180^{\circ} \mathrm{C}\right)$. Moreover, our $\mathrm{CG}$ macrostructures have high density and a compressive strength of $361 \mathrm{MPa}$ (over 6 times that of conventional graphite products), and thus can be expected to create new applications for graphene.

\section{FABRICATION OF COMPACT GRAPHENE}

\section{A. Preparation of graphite oxide}

GO was prepared by a modified Hummers method [12]. While maintaining agitation, expandable graphite powder $(2 \mathrm{~g})$ and sodium nitrate $(1 \mathrm{~g})$ were mixed with sulfuric acid $(46 \mathrm{ml}$, $98 \mathrm{wt} \%)$ in an ice bath, and potassium permanganate $(6 \mathrm{~g})$ was slowly added to prevent the temperature from exceeding $20^{\circ} \mathrm{C}$. The reaction was kept at $35 \pm 1^{\circ} \mathrm{C}$ for $8 \mathrm{~h}$ with gas release, and then deionized water $(92 \mathrm{ml})$ was gradually added, bringing about violent effervescence. The temperature of the water bath was increase to $98{ }^{\circ} \mathrm{C}$ and the reaction was maintained for 15 min in order to increase the oxidation degree of the GO product. The resultant bright-yellow suspension was diluted with deionized $(280 \mathrm{ml})$ water and further treated with a $\mathrm{H}_{2} \mathrm{O}_{2}$ solution (6 $\mathrm{ml}, 30 \%)$. The product was separated by centrifugation, washed with $5 \%$ chlorhydric acid $(\mathrm{HCl})$ solution for 7 times until sulphate could not be detected with $\mathrm{BaCl}_{2}$, then also washed 7 times with distilled water aiming at removing chloride ion. Then it was dried in an air oven at $60{ }^{\circ} \mathrm{C}$ overnight.

\section{B. Fabrication of compact graphene}

Colloidal dispersion of GO was hydrothermally reduced in $\mathrm{dH}_{2} \mathrm{O}$ with different amounts of ammonia. In each case, 57

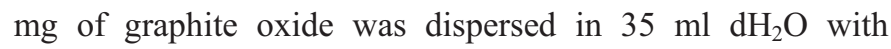
ammonia $(25-28 \mathrm{v} / \mathrm{v} \%)$ followed by sonication for $15 \mathrm{~min}$. Dark brown colloidal dispersion was transferred to a sealed reactor which was then heated to $180{ }^{\circ} \mathrm{C}$ for $15 \mathrm{~h}$. After heat treatment, the reactor was left to room temperature. In order to fabricate castings, plastic containers with complex shapes were designed. The reduced graphene suspension was moulded in the container and was treated in an air drier at $32{ }^{\circ} \mathrm{C}$ for at least $15 \mathrm{~h}$ to completely dry the casting.

\section{RESULtS}

The synthesis of CG is described as follows: Graphite oxide powder was dispersed in ultrapure water and sonicated, forming a brown colloidal aqueous dispersion of graphene oxide (GO) platelets. The colloid was transferred to a sealed reactor, ammonia or $\mathrm{NaOH}$ was added and the reactor heated sufficiently to remove many of the functional groups of GO, reducing GO into reduced graphene oxide (rGO) platelets, which we hereafter refer to as "graphene". Graphene gel was 
then taken out of the reactor and dried at a temperature slightly

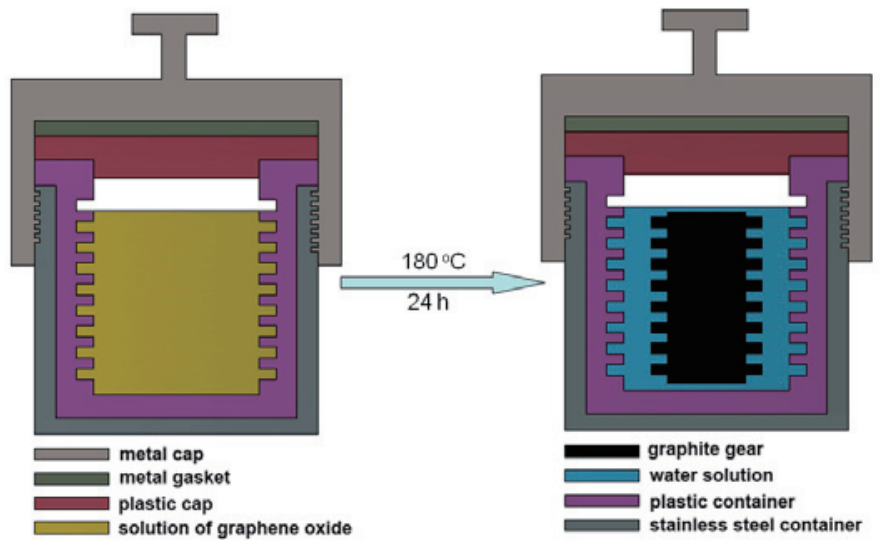

Fig. 1. Schematic diagram of graphene casting. G-O platelets are casted into a screw stem under the influence of temperature, pressure, and the container shape.

higher than room temperature. Fig. 1 illustrates the process of casting of such graphene platelets, where the dispersion of GO platelets was placed into a screw-like, sealed container and reduced at $180{ }^{\circ} \mathrm{C}$, after which it is black or dark grey. The resulting graphene gel was formed into a screw stem. For clarity, CG with the proper, excess, and no ammonia content were abbreviated as CGP $(\mathrm{pH}=10.1)$, CGE $(\mathrm{pH}=11.6)$, and CGN ( $\mathrm{pH}=5.5)$, respectively. When the wet gel was taken out of autoclave, and dried at temperature of $60{ }^{\circ} \mathrm{C}$ for $15 \mathrm{~h}$, the gel became a compact graphene which kept its original shape of wet gel (Fig. 2).

"Graphene castings" can be easily produced by this approach compared to other methods. Different castings were made, e.g., triangular prism, quadrangular prism, joint ring, crucible, screw stem, and gear, as shown in Fig. 3. The sizes of castings range from sub-millimeter to sub-meter, depending on the moulds and the concentrations of GO dispersion. In order to be transformed into malleable graphene gel, the requirement of lowest $\mathrm{GO}$ concentration should be $0.5 \mathrm{mg} / \mathrm{ml}$ [13].

In order to clarify the relationship between $\mathrm{pH}$ and mechanical property of CG, scanning electron microscopy (SEM) was applied to study the surface morphology and structure of cross-section (Fig. 4). Images of the surfaces of $\mathrm{CG}$ demonstrated the importance of $\mathrm{pH}$. Rugged CGN surface accompanied with pits throughout the surface (Fig. 4a). There were also many but smaller protuberances on the surface of CGE (Fig. 4c), which may imply the fact that a great deal of ammonia vapor had escaped from graphene gel during solidification. Contrarily, CGP appeared a smooth surface (Fig.

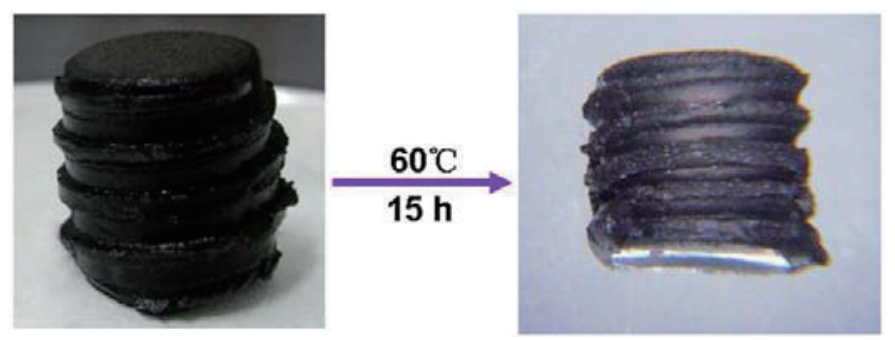

Fig. 2. The wet graphene gel can be shrinked into compact graphene after drying at temperature of $60^{\circ} \mathrm{C}$.
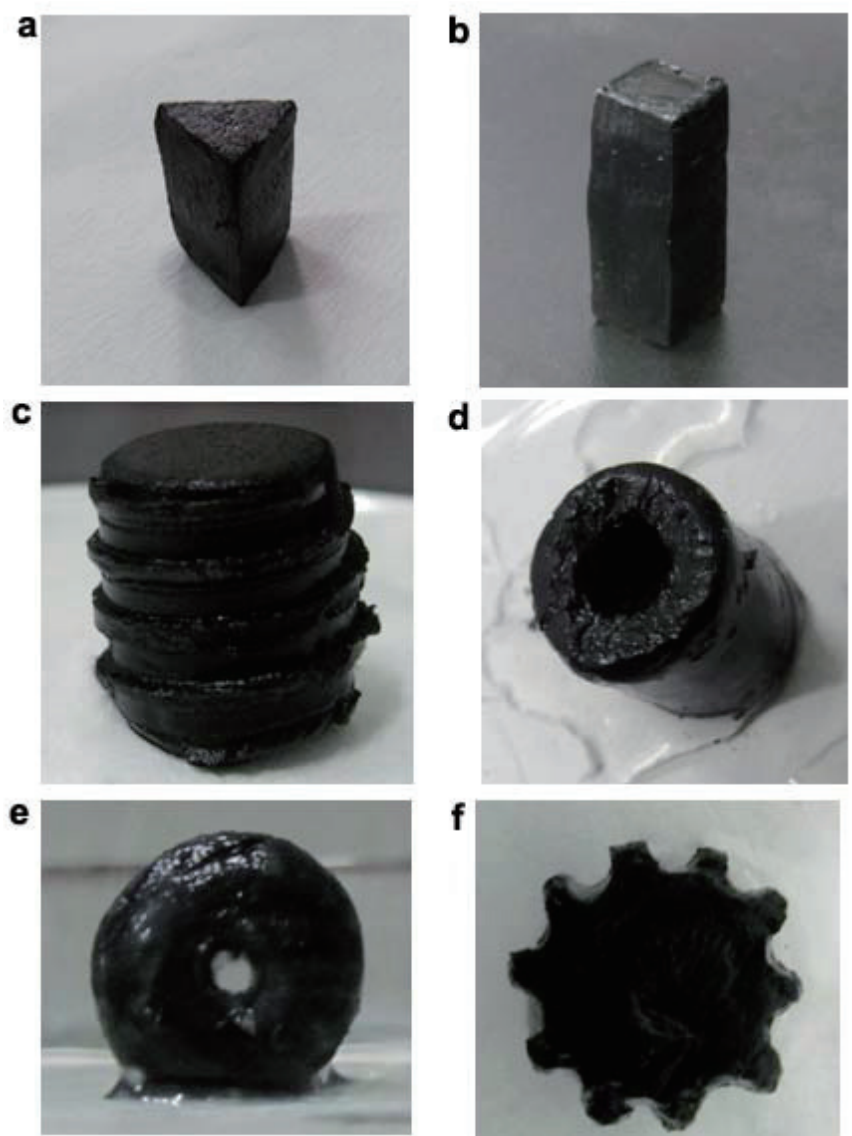

Fig. 3. Different castings of the graphene before drying. (a) triangular prism. (b) quadrangular prism. (c) screw stem. (d) crucible. (e) joint ring. (f) gear.

4e). Similar phenomenon was also observed in the digital photographs. Figs. $4 \mathrm{~b}$ and $4 \mathrm{~d}$ demonstrated that either shortage or excess of ammonia lead to the formation of holes and cracks within the $\mathrm{CG}$ during agglomeration of graphene sheets. These imperfections could decrease density, compressive strength and conductivity. A dense structure of CGP without apparent imperfection can be acquired with a proper $\mathrm{pH}$ (Fig. 4f). Comparisons among the cross-sections of the three CG further proved that the quantity of ammonia, in another word the $\mathrm{pH}$, played a critical role during the agglomeration of graphene sheets, and had further impact on the performance of CG.

During the repetitive synthesis of $\mathrm{CG}$, the impact of $\mathrm{pH}$ to the physico-chemical quality of castings has emerged. Relationships between compressive strength, conductivity and $\mathrm{pH}$ have then been studied. In this section, CG were obtained from graphene oxide dispersions with different $\mathrm{pH}$ values adjusted by ammonia ( $\mathrm{pH} 5.5 \sim 10.1$ ) or sodium hydroxide $(\mathrm{pH}$ 10.7 11.6). The details were shown in Table 1. The compressive strength of $\mathrm{CGN}(\mathrm{pH}=5.5,135 \mathrm{MPa})$ was apparently smaller than that of CGP ( $\mathrm{pH}=10.1,361 \mathrm{Mpa})$, indicating the contribution of proper $\mathrm{pH}$ to improve compressive strength. According to the results, the CG would achieve maximum compressive strength when the $\mathrm{pH}$ of GO dispersion was adjusted to 10 . Electrical conductivity of $\mathrm{CG}$ 
was found greatly affected by $\mathrm{pH}$ as well. The maximum conductivity for $\mathrm{CG}(\mathrm{pH}=10)$ is up to $7.6 \mathrm{~S} / \mathrm{cm}$, which was at least five times as great as that of CGN. And the value is very close to that of graphene foam [14].

In summary, a feasible method for $\mathrm{CG}$ fabrication has been developed, whose production showed that the $\mathrm{pH}$ of the graphene oxide dispersions a key factor which determined properties of its product. $\mathrm{pH}$ has a direct impact on surface topography, internal structure, thus electrical and mechanical
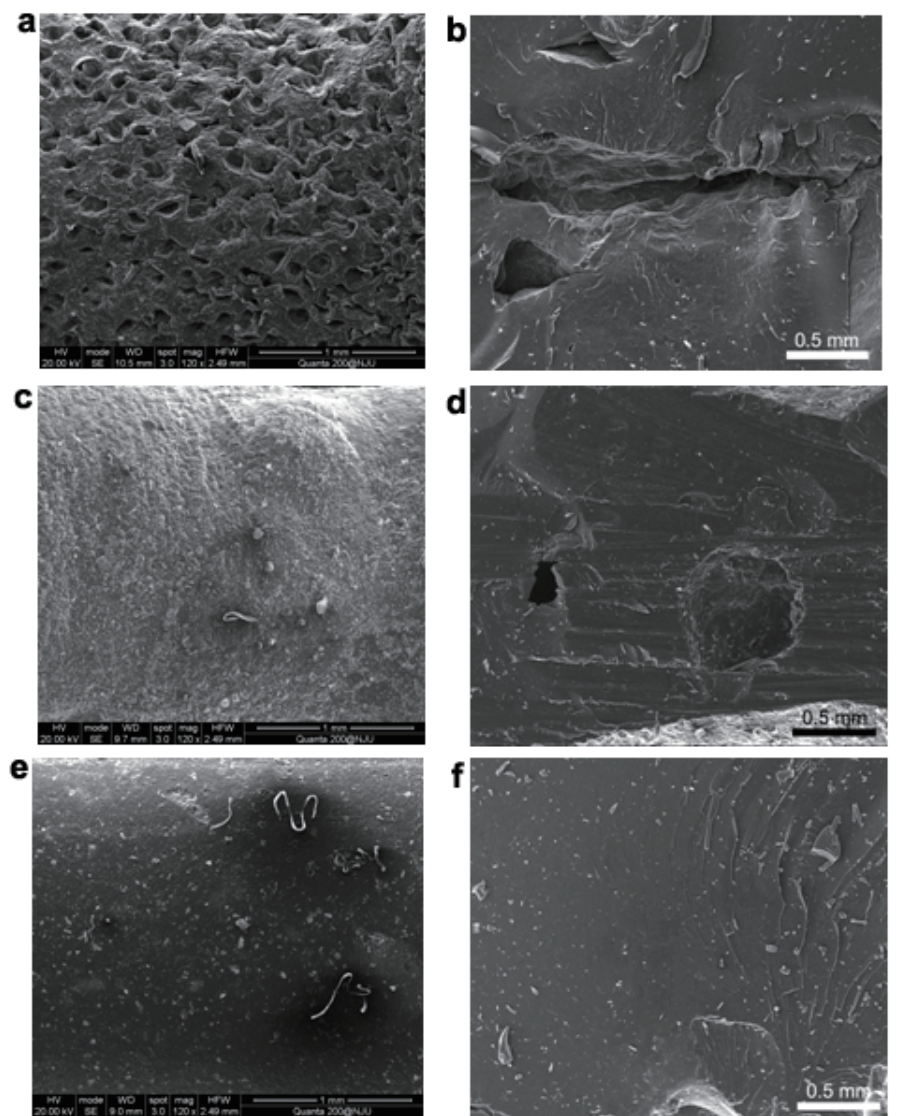

Fig. 4. SEM images of surface and cross-section of CG. (a) CGN, rough surface, holes could be observed. (b) CGN, holes and cracks inside. (c) CGE, rough surface, protuberances could be observed. (d) CGE, imperfections were observed. (e) CGP, smooth surface was produced when $\mathrm{pH}=10$. (f) CGP, graphene sheets combined solidly. No imperfection was found in this case.

properties of the CG. CG has the best structure and properties when the $\mathrm{pH}$ of reaction was adjusted to 10 , i.e., smooth surfaces, dense internal structures, good electrical conductivity, and high compressive strength. Even the density, compressive strength, and electrical conductivity have been further improved $\left(2.31 \mathrm{~g} / \mathrm{cm}^{3}, 361 \mathrm{MPa}\right.$, and $7.6 \mathrm{~S} / \mathrm{cm}$, respectively) by high temperature annealing at $900{ }^{\circ} \mathrm{C}$. All these parameters were far higher than conventional products, e.g. isotropic graphite. Meanwhile, the proposed method tended to be lowcost, environment-friendly, and energy-saving. CG have been proved a great potential to be applied to a wide range of fields from traditional graphite industry (graphite heaters, electrodes, and crystallizers) to aerospace, atomic energy and nuclear science. This work also pointed a new route to prepare highly
TABLE I. THE EFFECTS OF PH ON THE COMPRESSIVE STRENGTH AND ELECTRIC CONDUCTIVITY

\begin{tabular}{|c|c|c|}
\hline $\mathrm{pH}$ & $\begin{array}{c}\text { Compressive strength } \\
(\mathrm{MPa})\end{array}$ & $\begin{array}{c}\text { Electrical conductivity } \\
\left(\mathrm{S} \mathrm{cm}^{-1}\right)\end{array}$ \\
\hline 5.5 & 135 & 1.5 \\
\hline 7.7 & 150 & 2.7 \\
\hline 8.5 & 220 & 3.5 \\
\hline 9.4 & 248 & 6.8 \\
\hline 10.1 & 361 & 7.6 \\
\hline 10.7 & 30 & 2.1 \\
\hline 11.6 & 18 & 1.9 \\
\hline
\end{tabular}

compressed and super strong three-dimensional macrostructures based on graphene.

\section{ACKNOWLEDGMENT}

This work was supported by the National Basic Research Program of China (Grant No.2011CB707601 and No.2009CB623702), the National Natural Science Foundation of China (Nos 51071044, 60976003, 61006011 and 61106055), Specialized Research Fund for the Doctoral Program of Higher Education (Nos 20100092120021 and 20100092110014) Program for New Century Excellent Talents in University (No. NCEF-09-0293) and Open Research Fund of State Key Laboratory of Bioelectronics.

\section{REFERENCES}

[1] K.S. Novoselov, et al., "Electric field effect in atomically thin carbon films," Science, vol. 306, pp. 666-669, 2004.

[2] K.S. Novoselov, et al., "Two-dimensional gas of massless Dirac fermions in graphene," Nature, vol. 438, pp. 197-200, 2005.

[3] Y. Zhang, Y-W. Tan, H.L. Stormer, and P. Kim, "Experimental observation of the quantum Hall effect and Berry's phase in graphene," Nature, vol. 438, pp. 201-204, 2005.

[4] A.A. Balandin, et al., "Superior thermal conductivity of single-layer graphene," Nano Lett., vol. 8, pp. 902-907, 2008.

[5] R. Prasher, "Graphene spreads the heat," Science, vol. 328, pp. 185-186, 2010 .

[6] C. Lee, X.D. Wei, J.W. Kysar, and J. Hone, "Measurement of the elastic properties and intrinsic strength of monolayer graphene," Science, vol. 321, pp. 385-388, 2008.

[7] Y. Hernandez, et al., "High-yield production of graphene by liquidphase exfoliation of graphite," Nature Nanotech., vol. 3, pp. 563-568, 2008 .

[8] P.W. Sutter, J.I. Flege, and E.A. Sutter, "Epitaxial graphene on ruthenium," Nature Mater., vol. 7, pp. 406-411, 2008.

[9] W. Gao, L.B. Alemany, L.J. Ci, and P.M. Ajayan, "New insights into the structure and reduction of graphite oxide," Nature Chem., vol. 1, pp. 403-408, 2009.

[10] V.H. Pham, et al., "One-step synthesis of superior dispersion of chemically converted graphene in organic solvents," Chem. Commun., vol. 46, pp. 4375-4377, 2010.

[11] H.L.Wang, J.H. Robinson, X.1. Li, and H.J. Dai, "Solvothermal reduction of chemically exfoliated graphene sheets," J. Am. Chem. Soc., vol. 131, pp. 9910-9911, 2009.

[12] O.C. Compton, and S.T. Nguyen, "Graphene oxide, highly reduced graphene oxide, and graphene: versatile building blocks for carbonbased materials," Small, vol. 6, pp. 711-723, 2010. 
[13] Y.X. Xu, K.X. Sheng, C. Li, and G.Q. Shi, "Self-Assembled graphene hydrogel via a one-step hydrothermal process," ACS Nano, vol. 4, pp. 4324-4330, 2010.

[14] Z.P. Chen, et al., "Three-dimensional flexible and conductive interconnected graphene networks grown by chemical vapour deposition," Nature Mater., vol. 10, pp. 424-428, 2011. 